\title{
Analysis and control design of two cascaded boost converter
}

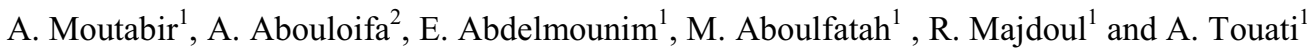 \\ ${ }^{1}$ L.A.S.T.I, University HASSAN 1er, Faculty of sciences and technology of Settat, Morocco \\ ${ }^{2}$ L.T.I, University HASSAN II, Faculty of Sciences Ben M'sik Casablanca, Morocco
}

\begin{abstract}
This work aims to study a cascade of two BOOST converters. First, a non- linear model of the whole controlled system is developed. Then, a robust non-linear controller of currents is synthesized using a backstepping design technique. A formal analysis based on Lyapunov stability and average theory is developed to describe the control currents loops performances. A classical PI controller is used for the voltages loops. The study of the stability of the system will also be discussed. Simulated results are displayed to validate the feasibility and the effectiveness of the proposed strategy.
\end{abstract}

\section{Introduction}

The DC / DC converters have become an essential component of industrial and military applications over the past decades. Thanks to their efficiency increasingly high, their size, their weight and reduced cost, they have replaced conventional linear power supplies, even at low power levels [1].

Obtaining a high voltage is needed in some applications which the input supply is low such as photovoltaic panel or fuel cell.

The problem can be handled either by using a simple step-up converter with high duty cycle or by using cascaded converters. The use of a single stage in performing this conversion ratio will imply working with high duty cycles and therefore will increase the losses and reduce the voltage conversion ratio. Moreover, the use of an individual power converter with a high duty cycle to obtain high voltage conversion ratios has some design limitations due to the finite commutation times of the power devices and the size of the passive elements. If galvanic isolation is not required, the cascade connection of two boost converters can be a good alternative to obtain high voltage step-up ratios.

Some previous works have presented a studies of cascaded converters, such as combination of an interleaved converter and inverter three levels [2], and a comparison between static and dynamic performances of a Z-source converter and dual-stage boost converter [3]. In this article, a study of two cascaded boost converter will be presented.
A control strategy is developed that simultaneously deals with the two cascaded boost. The control strategy is featured by its multi-loops nature. First, the two current loops are designed using the backstepping technique [4 ]. Then, two others loops are designed to regulate the output voltage and the voltage between the two boost converters using the classical PI regulator.

Additional actions are designed to dominate the disturbing effects while preserving the closed-loop system stability. It will be formally proved that the robust multi-loop controller thus obtained stabilizes (globally and asymptotically) the controlled system and ensures quite interesting tracking properties [5].

These theoretical results are obtained making judicious use of adequate control theory tools including averaging theory and Lyapunov stability [6] . The paper is organized as follows: the system under study (i.e. the DC-DC cascaded boost converter) is modeled and given a state space representation in Section2; the controllers design and the closed-loop system analysis are dealt with in Section3; the controller performances are illustrated through numerical simulations in Section4.

\section{Modeling the two cascaded boost converter}

The circuit in figure 1 consists of two cascaded Boost converter. The boost converters are according to the known pulse wide modulation (PWM) principle. 


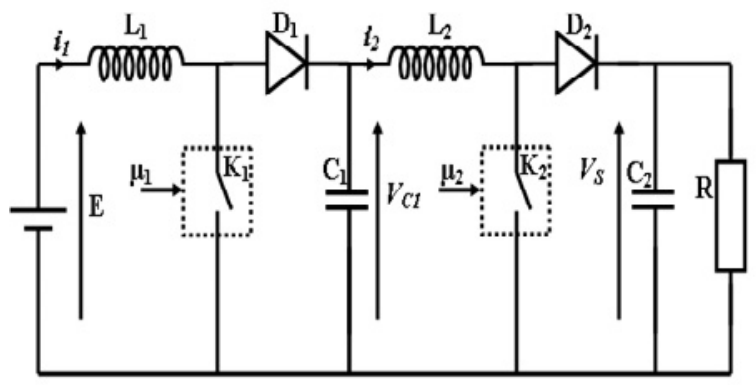

Fig. 1. Two cascaded boost converter.

The used parameter values of the present converter are depicted in Table 1.

Table 1. The used parameter values for the converter.

\begin{tabular}{|c|c|}
\hline Prameter & Value \\
\hline Inductors & $\begin{array}{c}L_{1}=35 \mu \mathrm{H} \\
L_{2}=100 \mu \mathrm{H}\end{array}$ \\
\hline Capacitors & $\begin{array}{c}C_{1}=4 \mathrm{mF} \\
C_{2}=600 \mu \mathrm{F}\end{array}$ \\
\hline Frequency & $10 \mathrm{kHz}$ \\
\hline Input voltage & $E=12 \mathrm{~V}$ \\
\hline Load resistor & $R=10 \Omega$ \\
\hline Swiches & IGBT \\
\hline
\end{tabular}

\subsection{Switched model}

By applying standard Kirchhof Voltage Law (KVL) and Kirchhof Current Laws (KCL) to the circuit depicted in Figure 1, the two cascaded Boost converter can be represented by the following differential equations:

$L_{1} \frac{d i_{1}}{d t}=E-\left(1-\mu_{1}\right) V_{c 1}$

$C_{1} \frac{d V_{c 1}}{d t}=\left(1-\mu_{1}\right) i_{1}-i_{2}$

$L_{2} \frac{d i_{2}}{d t}=V_{c 1}-\left(1-\mu_{2}\right) V_{s}$

$C_{2} \frac{d V_{s}}{d t}=\left(1-\mu_{2}\right) i_{2}-\frac{V_{s}}{R}$

Where:

- $\quad i_{1}$ and $i_{2}$ are respectively the currents in inductors $L_{1}$ and $L_{2}$,

- $\quad V_{c 1}$ denotes the voltage in capacitor $C_{1}$ and $V_{s}$ is the output voltage.

- $\quad \mu_{1}$ and $\mu_{2}$ are denoted the duties ratio functions. $\mu_{1}=\left\{\begin{array}{l}1 \text { if } K_{1} \text { is } O N \\ 0 \text { if } K_{1} \text { is } O F F\end{array} \quad\right.$ and $\quad \mu_{2}=\left\{\begin{array}{l}1 \text { if } K_{2} \text { is } O N \\ 0 \text { if } K_{2} \text { is } O F F\end{array}\right.$

\subsection{Averaged model}

This model is defined as follows:

$$
\begin{aligned}
& \dot{x}_{1}=-\frac{1-u_{1}}{L_{1}} x_{2}+\frac{E}{L_{1}} \\
& \dot{x}_{2}=\frac{1-u_{1}}{C_{1}} x_{1}-\frac{1}{C_{1}} x_{3} \\
& \dot{x}_{3}=\frac{1}{L_{2}} x_{2}-\frac{1-u_{2}}{L_{2}} x_{4} \\
& \dot{x}_{4}=\frac{1-u_{2}}{C_{2}} x_{3}-\frac{1}{R C_{2}} x_{4}
\end{aligned}
$$

In the above model, $x_{1}, x_{2}, x_{3} x_{4}$ are denoted respectively the averages currents and voltages $i_{1}, V_{c 1}, i_{2}, V_{s}$ and $\left(u_{1}, u_{2}\right)$ represents the averages duties ratio function $\left(\mu_{1}, \mu_{2}\right)$.

\subsection{Equilibrium points}

The equilibrium point can be obtained by forcing the time derivative of the state variables of the reduced order model to be null.

$$
\dot{x}=0 \Rightarrow\left\{\begin{aligned}
x_{1}^{0} & =\frac{1}{\left(1-U_{1}^{0}\right)^{2}\left(1-U_{2}^{0}\right)^{2}} \frac{E}{R} \\
x_{2}^{0} & =\frac{E}{1-U_{1}^{0}} \\
x_{3}^{0} & =\frac{E}{R\left(1-U_{1}^{0}\right)\left(1-U_{2}^{0}\right)^{2}} \\
x_{4}^{0} & =\frac{1}{\left(1-U_{1}^{0}\right)\left(1-U_{2}^{0}\right)} E
\end{aligned}\right.
$$

The equilibrium point is noted:

$X^{0}=\left(\begin{array}{llll}x_{1}^{0} & x_{2}^{0} & x_{3}^{0} & x_{4}^{0}\end{array}\right)$

From the equilibrium point, the output power is equal to the input power and that the input voltage in a boost converter is less than its output voltage

\subsection{Stability analysis}

In order to study the stability of the system, the nonlinear model (2a)-(2d) is linearized around the equilibrium point $X^{0}$ given by (3e). The stability of the linearized system can be studied by using the Jacobian matrix $\mathrm{A}$ and evaluating it at the equilibrium point $X^{0}$. This matrix can be expressed as follows: 
$A=\frac{\partial f}{\partial x} \mid \begin{aligned} & U=U^{0} \\ & X=X^{0}\end{aligned}$

$\mathrm{A}=\left(\begin{array}{cccc}0 & -\frac{\left(1-u_{1}^{0}\right)}{L_{1}} & 0 & 0 \\ \frac{\left(1-u_{1}^{0}\right)}{C_{1}} & 0 & -\frac{1}{C_{1}} & 0 \\ 0 & \frac{1}{L_{2}} & 0 & -\frac{\left(1-u_{2}^{0}\right)}{L_{2}} \\ 0 & 0 & \frac{\left(1-u_{2}^{0}\right)}{C_{2}} & -\frac{1}{R C_{2}}\end{array}\right)$

The local stability analysis of the system can be carried out by using the characteristic polynomial equation: $p(s)=\operatorname{det}(\mathrm{A}-s \mathrm{I})=0$ of the linearized system, where I is the unitary matrix.

Using Routh-Hurwitz criterion, four conditions for stability are obtained.

If we consider the choice equilibrium point:

$X^{0}=\left(\begin{array}{llll}7,5 & 18 & 5 & 30\end{array}\right)^{T}$

$U=(1 / 3 \quad 0,4)^{T}$

$p(s)$ can be written in the following form:

$\operatorname{det}(s I-A)=s^{4}+166,7 s^{3}+3174 \cdot 10^{3} s^{2}+546,2 \cdot 10^{6} s$

$$
+39,7 \cdot 10^{12}=0
$$

The roots are located in the left plane and hence, the system is asymptotically stable.

We have:

$\mathrm{B}=\left(\begin{array}{ll}\frac{\partial f_{1}}{\partial u_{1}} & \frac{\partial f_{1}}{\partial u_{2}} \\ \frac{\partial f_{2}}{\partial u_{1}} & \frac{\partial f_{2}}{\partial u_{2}} \\ \frac{\partial f_{3}}{\partial u_{1}} & \frac{\partial f_{3}}{\partial u_{2}} \\ \frac{\partial f_{4}}{\partial u_{1}} & \frac{\partial f_{4}}{\partial u_{2}}\end{array}\right)=\left(\begin{array}{cc}\frac{x_{2}^{0}}{L_{1}} & 0 \\ \frac{x_{1}^{0}}{C_{1}} & 0 \\ 0 & \frac{x_{4}^{0}}{L_{2}} \\ 0 & \frac{x_{3}^{0}}{C_{2}}\end{array}\right)$

$\mathrm{C}=\left(\begin{array}{llll}\frac{\partial y}{\partial x_{1}} & \frac{\partial y}{\partial x_{2}} & \frac{\partial y}{\partial x_{3}} & \frac{\partial y}{\partial x_{4}}\end{array}\right)=\left(\begin{array}{llll}0 & 0 & 0 & 1\end{array}\right)$

$y=x_{4}$

The transfer function defined by:

$H_{s}(s)=C(s I-A)^{-1} B+D$

We can justify that the system is a non- minimum phase because it contains some zeros dynamics

The output is given by:

$$
Y(s)=\frac{N_{1}(s)}{\operatorname{det}(s I-A)} U_{1}(s)+\frac{N_{2}(s)}{\operatorname{det}(s I-A)} U_{2}(s)
$$

Then:

$N_{1}(s)=-(5860 s+122.45) 10^{12}$
$N_{2}(s)=\left(0,0083 s^{3}+7800 s^{2}-69000 s-2481010^{6}\right) 10^{6}$

\section{Control design}

\subsection{Control objectives}

There are four operational control objectives:

(i) Current regulation: the input current $x_{1}$ (respectively the current $x_{3}$ in inductor $L_{2}$ ) must track, as closely as possible, a given reference signal $x_{1}^{*}$ ( respectively $x_{3}^{*}$ ).

(ii) Controlling the voltage $x_{2}$ making it track a given reference signal $x_{2}^{*}$.

(iii) Regulating the output voltage $x_{4}$ to a reference value $x_{4}^{*}$.

(iv) Ensure the global stability of the system.

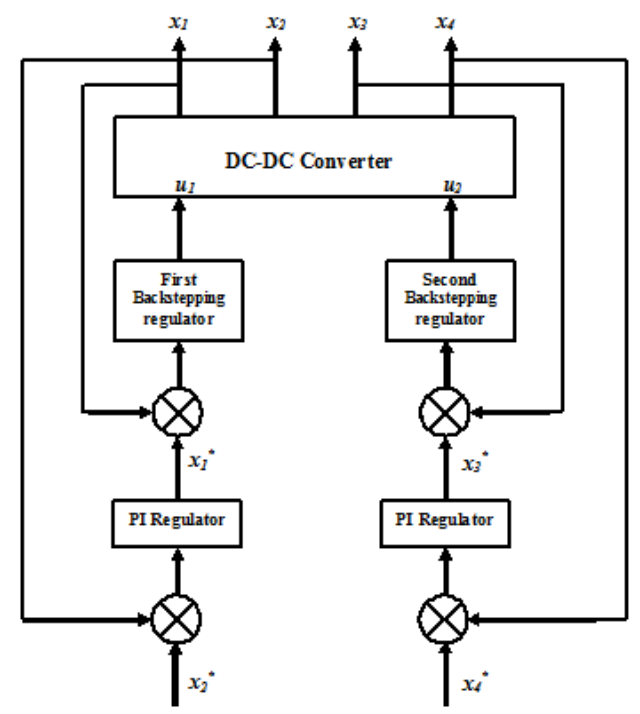

Fig. 2. Regulators based structure.

As shown in figure 2 we define two current loops ( inner loop) and two voltage loops ( outer loop).

\subsection{Inner loop}

Consider the tracking errors $z_{1}$ and $z_{3}$ defined by:

$z=\left(\begin{array}{l}z_{1} \\ z_{3}\end{array}\right)=\left(\begin{array}{l}L_{1}\left(x_{1}-x_{1}^{*}\right) \\ L_{2}\left(x_{3}-x_{3}^{*}\right)\end{array}\right)$

Its derivative is given by:

$\dot{z}=\left(\begin{array}{l}\dot{z}_{1} \\ \dot{z}_{3}\end{array}\right)=\left(\begin{array}{l}L_{1}\left(\dot{x}_{1}-\dot{x}_{1}^{*}\right) \\ L_{2}\left(\dot{x}_{3}-\dot{x}_{3}^{*}\right)\end{array}\right)$

Consider the quadratic Lyapunov function:

$V=\frac{1}{2} z^{T} z$ 
Its derivative is done:

$\dot{V}=\frac{1}{2}\left(\dot{z}^{T} z+z^{T} \dot{z}\right)=z_{1} \dot{z}_{1}+z_{3} \dot{z}_{3}$

To get a stabilizing control laws, the time-derivative $\dot{V}$ must be a negative definite function of $z_{1}$ and $z_{3}$. Then $\dot{V}$ is chosen :

$\dot{V}=-k_{1} z_{1}^{2}-k_{3} z_{3}^{2}<0$

Where $k_{1}$ and $k_{3}$ are the constants positives parameters synthesis of the controllers.

This choice implies that:

$\left\{\begin{array}{l}\dot{z}_{1}=-k_{1} z_{1} \\ \dot{z}_{3}=-k_{3} z_{3}\end{array}\right.$

We replace $\dot{z}_{1}$ and $\dot{z}_{3}$ in (4), we obtain:

$$
\left\{\begin{array}{l}
-k_{1} z_{1}=L_{1}\left(\dot{x}_{1}-\dot{x}_{1}^{*}\right) \\
-k_{3} z_{3}=L_{2}\left(\dot{x}_{3}-\dot{x}_{3}^{*}\right)
\end{array}\right.
$$

Substituting $\dot{x}_{1}$ and $\dot{x}_{3}$ respectively by the relationships of the expressions (2a) and (2c), then:

$\left\{-k_{1} z_{1}=E-\left(1-u_{1}\right) x_{2}-L_{1} \dot{x}_{1}^{*}\right.$

$\left\{-k_{3} z_{3}=x_{2}-\left(1-u_{2}\right) x_{4}-L_{2} \dot{x}_{3}^{*}\right.$

Then the control laws to satisfy a stabilization are defined by:

$\left\{\begin{array}{l}u_{1}=1+\frac{L_{1} \dot{x}_{1}^{*}-k_{1} z_{1}-E}{x_{2}} \\ u_{2}=1+\frac{L_{2} \dot{x}_{3}^{*}-k_{3} z_{3}-x_{2}}{x_{4}}\end{array}\right.$

The properties of these control laws are summarized in the proposition 1 .

Proposition1. Consider the system composed of the currents equations (2a) and (2c), and the control laws (15) and (16) where $k_{1}>0$ and $k_{3}>0$ are arbitrarily chosen by the user. If the references $x_{1}^{*}$ and $x_{3}^{*}$ and their first time derivatives are available, then one has the following properties:

- $\quad$ The current loop undergoes the equations (9) and (10) with (5). As $k_{1}$ and $k_{3}$ are positives, these equations are exponentially stables.

- $\quad$ The input current and the current in inductor $L_{2}$ tends to their references.

\subsection{Outer loop}

The aim is now to design a tuning laws for the $x_{1}^{*}$ and $x_{3}^{*}$.

\subsubsection{Relationship between $x_{1}^{*}$ and $x_{2}$}

The first step in designing such a loop is to establish the relation between $x_{1}^{*}$ (control input current) and the output voltage $x_{2}$.

Using the equation (2b) and replacing $u_{1}$ by (15) and $x_{1}$, $x_{3}$ by the relations deduced for (4), we obtain:

$$
2 C_{1} x_{2} \dot{x}_{2}=2\left(k_{1} z_{1}+E-L_{1} \dot{x}_{1}^{*}\right)\left(\frac{z_{1}}{L_{1}}+x_{1}^{*}\right)-2 x_{2}\left(\frac{z_{3}}{L_{2}}+x_{3}^{*}\right)
$$

If we consider: $y_{2}=x_{2}^{2}$ then: $\quad \dot{y}_{2}=2 x_{2} \dot{x}_{2}$

$C_{1} \dot{y}_{2}=2\left(k_{1} z_{1}+E-L_{1} \dot{x}_{1}^{*}\right)\left(\frac{z_{1}}{L_{1}}+x_{1}^{*}\right)-2 x_{2}\left(\frac{Z_{3}}{L_{2}}+x_{3}^{*}\right)$ Finally:

$\dot{y}_{2}=\frac{2 E}{C_{1}} x_{1}^{*}+\frac{2 z_{1} k_{1}}{C_{1}} x_{1}^{*}-\frac{L_{1}}{C_{1}} \frac{d}{d t}\left(x_{1}^{* 2}\right)-\frac{2 z_{1}}{C_{1}} \dot{x}_{1}^{*}+X(x, t)$

With:

$$
X(x, t)=\frac{1}{C_{1}}\left(-2 \sqrt{y_{2}} x_{3}^{*}+\frac{2 k_{1}}{L_{1}} z_{1}^{2}+\frac{2 E}{L_{1}} z_{1}-\frac{2 z_{3}}{L_{2}} \sqrt{y_{2}}\right)
$$

Proposition 2. Consider the system described by (2a) and (2b) together with the control law (15). Under the same assumptions as in Proposition 1, one has the following properties:

(i) The output voltage $x_{2}$ varies, in response to the reference, according to the equation (17).

The squared voltage $y_{2}=x_{2}^{2}$ varies, in response to the reference, according to the equation (18) with (19).

\subsubsection{Controller PI design}

Note that the model (18) can be seen as an integrator disturbed by the signal $\mathrm{p}(\mathrm{t})$. The development of the controller is based on the following average model:

$\dot{\bar{y}}_{2}=\alpha \bar{x}_{1}^{*}+\bar{p}(t)$

with: $\alpha=\frac{2 E}{C_{1}}$

The system (20) can be stabilized using a simple PI corrector whose transfer function is given by:

$C(s)=k_{p 2}+\frac{k_{i 2}}{s}$.

The first derivative of the reference $x_{1}^{*}$ must be available

(Proposition 1), the low pass filter is adopted, then: 
$x_{1}^{*}=\left[k_{p 2}\left(y_{2}^{*}-y_{2}\right)+k_{i 2} \int\left(y_{2}^{*}-y_{2}\right) d t\right] \frac{b}{s+b}$

Then:

$x_{1}^{*}=\frac{b}{s+b}\left(k_{p 2} z_{2}+k_{i 2} z_{22}\right)$

With: $\left\{\begin{array}{l}z_{2}=y_{2}^{*}-y_{2} \\ z_{22}=\int z_{2} d t\end{array}\right.$

The block diagram of the controlled system is shown in Figure 3.

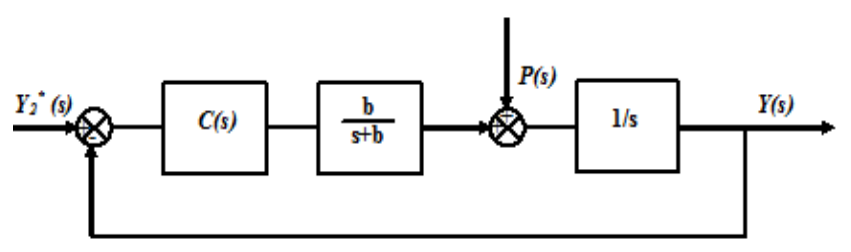

Fig. 3. Voltage equivalent loop.

In the closed loop, the output signal y depends on the reference and the disturbance signal $p(t)$ using the following equation:

$Y_{2}(s)=F(s) Y_{2}^{*}(s)+G(s) P(s)$

With:

$F(s)=\frac{b\left(k_{i 2}+k_{p 2} s\right)}{s^{3}+b s^{2}+k_{p 2} b s+k_{i 2} b}$

$G(s)=\frac{s(s+b)}{s^{3}+b s^{2}+k_{p 2} b s+k_{i 2} b}$

Using Routh-Hurwitz criterion, the conditions for stability are given by:

$\left\{\begin{array}{c}b>0 \\ k_{i 2}>0 \\ k_{p 2} b>k_{i 2}\end{array}\right.$

The transfer function $F(s)$ shows that the regulator guarantees perfect tracking $\lim _{t \rightarrow+\infty}\left(y_{2}^{*}-y_{2}\right)=0$ and $G(s)$ guarantees a disturbance rejection because it contains a derivative effect.

The parameters values chosen are depicted in Table 2 .

The BODE diagram in figure 4 shows the frequency behavior of $F(s)$.

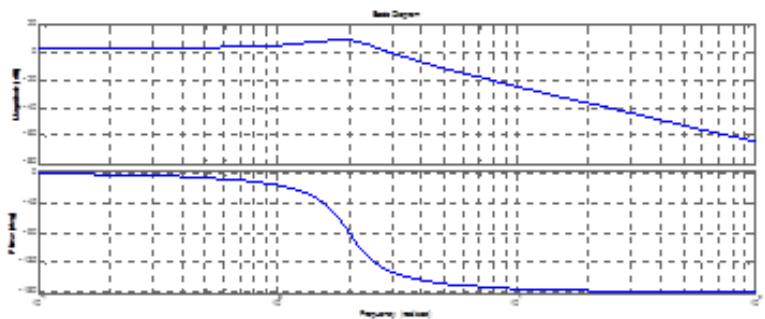

Fig. 4. Bode diagram of $F(s)$.

\subsubsection{Relationship between $x_{3}^{*}$ and $x_{4}$ :}

Proceed by the same approach that paragraph 3.3.2, we obtain:

$\dot{y}_{4}=\frac{2}{C_{2}}\left[\left(k_{3} z_{3}+x_{2}-L_{2} \dot{x}_{3}^{*}\right)\left(\frac{z_{3}}{L_{2}}+x_{3}^{*}\right)-\frac{2}{R} y_{4}\right]$

$x_{3}^{*}=\left[k_{p 4}\left(y_{4}^{*}-y_{4}\right)+k_{i 4} \int\left(y_{4}^{*}-y_{4}\right) d t\right] \frac{b^{\prime}}{s+b^{\prime}}$

$x_{1}^{*}=\frac{b}{s+b}\left(k_{p 2} z_{2}+k_{i 2} z_{22}\right)$

With: $\left\{\begin{array}{l}z_{2}=y_{2}^{*}-y_{2} \\ z_{22}=\int z_{2} d t\end{array}\right.$

\section{Simulations}

In order to verify the theoretical results predicted in Sections 2 and 3, the two cascaded boost converter has been simulated by using SIMPOWER of MatlabSimulink software with the set of parameter values depicted in Tables 1 and 2 .

Table 2. Parameter values for the simulations.

\begin{tabular}{|c|c|}
\hline Parameters & Values \\
\hline$k_{1}, k_{3}$ & 200 \\
\hline Integral: $k_{i 2}, k_{i 4}$ & 4 \\
\hline Proportional: $k_{p 2}, k_{p 4}$ & 2 \\
\hline Low filter: $b, b^{\prime}$ & 3 \\
\hline Reference: $x_{2}^{*}$ & $\left\{\begin{array}{c}\text { Initial value: } 18 \\
\text { Final value: } 36\end{array}\right.$ \\
\hline Reference: $x_{4}^{*}$ & $\left\{\begin{array}{c}\text { Initial value: } 30 \\
\text { Final value: } 60\end{array}\right.$ \\
\hline
\end{tabular}

The converter has been simulated to check the previous stability conditions.

The figures 5-6 and 7-8 present respectively the forms of currents and voltages. We observe that the tracking is satisfied and the stability is guaranteed.

The forms of the errors are showed in figures 9 and 10 . 


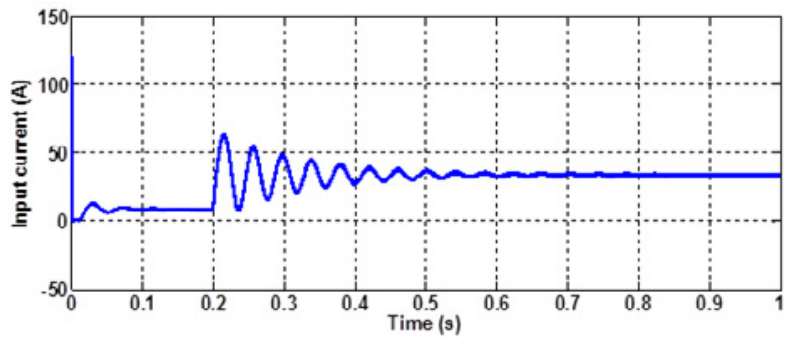

Fig. 5. Input current.

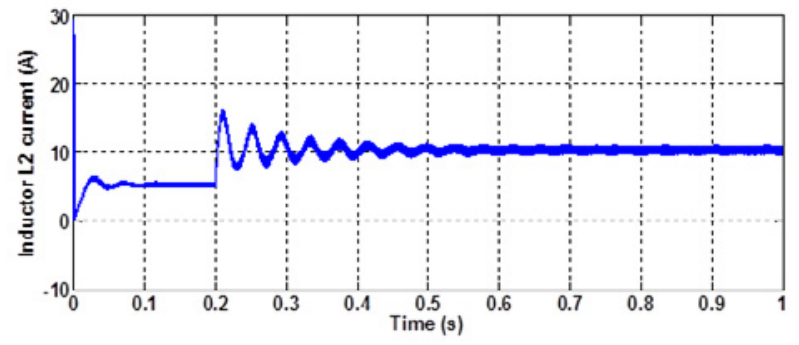

Fig. 6. Inductor $L_{2}$ current.

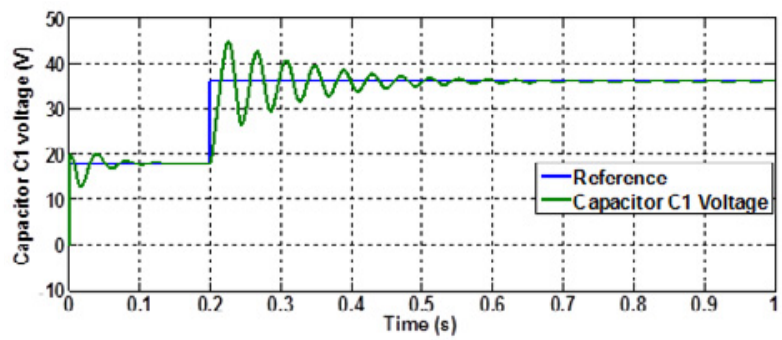

Fig. 7. Capacitor $C_{1}$ voltage.

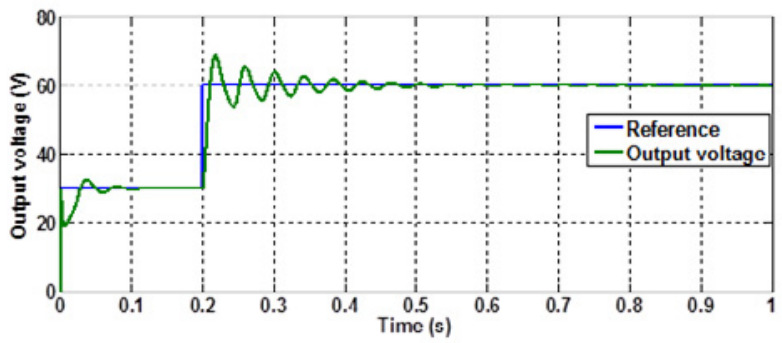

Fig. 8. Output voltage.

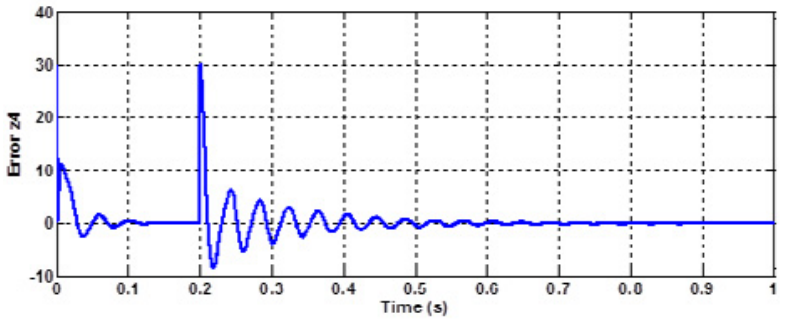

Fig. 9. Tracking error $z_{4}$.

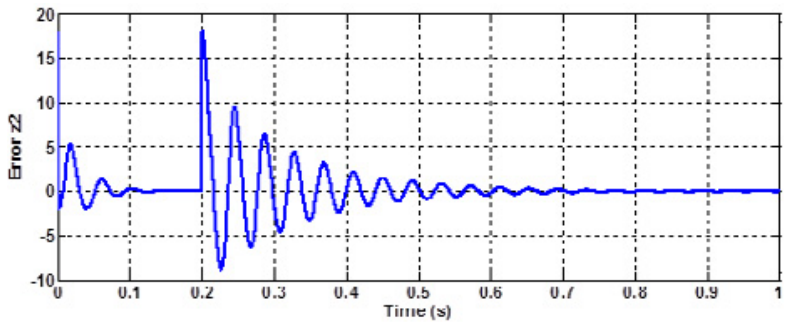

Fig. 10. Tracking error $z_{2}$.

\section{Conclusion}

In some applications, high conversion ratios are needed. Different techniques and converter topologies can be obtained to handle this problem. In this paper, the two cascaded boost converter has been studied for his potential use in this kind of applications. It has been analyzed theoretically and by numerical simulations using Matlab software. The dynamical models and stability analysis of this system has been presented. The controller design have been studied also. The results of simulations have been satisfied.

\section{References}

1. H. Sira-Ramirez, M. Garcia-Esteban, A.S.I. Zinober, PWM DC-DC power converter .Int. J. E 65, 205 (1996)

2. A. Shahin, B. Huang, J.P. Martin., S. Pierfederici,. B. Davat.New non linear control strategy for $D C / D C$ converter E.C.M 51, 56 (2010)

3. A. Elaroudi, R. Haroun, A. Cid-Pastor, A. Kouzou, L. Martinez-Salamero.,Z-source and dual-stage boost comparison Energie Procedia 42, 587 (2013)

4. J. Alvarez-Ramirez, G. Espinosa-Perez, D. NoriegaPineda. Banaszak Backstepping approach.IEEE., 190 (2001)

5. A. Elmaguiri, F. Giri, A. Abouloifa, F.Z. Chaoui. Robust control , Elsevier (2010)

6. H. Elfadil, F. Giri, F.Z. Chaoui., M. Haloua, H. Ouadi, Non-linear and adaptive control. IEEE $\mathbf{4 2 , 4 4 7 5}$ (2003) 